Trauma Berufskrankh 2014 · 16[Suppl 3]:297-299 DOI 10.1007/s10039-014-2062-x

Online publiziert: 19. April 2014

(c) Springer-Verlag Berlin Heidelberg 2014

\title{
B. Bickert
}

Sektion Handchirurgie, Klinik für Hand-, Plastische und Rekonstruktive Chirurgie, BG-Klinik Ludwigshafen

\section{Knocheninfekte an der Hand}

malisieren, während die Knochen im Verlauf von Monaten völlig destruiert werden.

\section{Diagnostik}

Bei akuten Infekten an der Hand (innerhalb von 1 bis 2 Wochen nach auslösendem Trauma) rechtfertigt schon allein der Verdacht eines Knochen- oder Gelenkinfekts die Operationsindikation. Eine umfangreiche Diagnostik ist hier meist nicht erforderlich. Umgekehrt sollte die Indikation zur konservativen Therapie, also trotz des Verdachts nicht zu operieren, sehr sorgfältig und sehr kritisch gestellt, begründet und dokumentiert werden, da der Schaden eines verschleppten Knochen- oder Gelenkinfekts gravierender ausfallen kann als es der einer vielleicht unnötigen Operation gewesen wäre.

Röntgenaufnahmen der Hand oder des Handgelenks sind unabdingbar. Einerseits können Osteolysen den Hinweis auf ein schon länger schwelendes Infektgeschehen liefern. Andererseits sollte nach röntgendichten Fremdkörpern gesucht werden. Bei Osteosynthesen würde eine Instabilität den Infektverdacht erhärten. Bei unklaren, insbesondere länger bestehenden Beschwerden kann sich in der Magnetresonanztomographie (MRT) ein Knochenödem als Hinweis auf eine Osteitis ergeben. Ein sehr aussagekräftiges Verfahren ist auch das SPECT/CT, bei dem die nuklearmedizinische SPECT („single photon emission computed tomography") mit dem Computertomogramm (CT) des Knochens kombiniert wird [3].

\section{Bakteriologie}

Beweisend für einen Knocheninfekt ist der Keimnachweis in Kombination mit dem histopathologischen Befund. Sollte bei einer operativen Revision Eiter gefunden werden, mag aus einem bakteriologischen Abstrich ein aussagekräftiger Keimnachweis gelingen, was aber fast nie der Fall ist. Wesentlich zuverlässiger sind multiple Probebiopsien vom Knochen, die nicht nur histopathologisch, sondern auch bakteriologisch befundet werden [5]

\section{Differenzialdiagnose}

Weniger bei Knochen- als vielmehr bei Weichteilinfekten spielte in den letzten Jahren die Folge einer Octenidinspülung (z. B. Octenisept $t^{\circledast}$ ) nach Handverletzungen eine erhebliche differenzialdiagnostische Rolle, da die resultierende Weichteilreaktion manchmal über mehrere Wochen einen Infekt imitieren kann [2]. Als antibakterielle Spüllösung sollten deshalb an der Hand ausschließlich isotone Elektrolytlösungen (z. B. Ringer-Lösung) eingesetzt werden.

\section{Therapie}

In vielen Fällen von Knocheninfekten verbietet sich ein einzeitiges Vorgehen (- Abb. 1). Stattdessen erfolgt in einem ersten oder auch in mehrfachen Eingriffen das ausgiebige knöcherne Débridement mit temporärer Stabilisierung und Einlage eines antibiotikahaltigen Spacers [4]. Da ein Knocheninfekt nur unter einer suffizienten Weichteilbedeckung aushei- 


\section{Hand- und Unterarminfektionen}
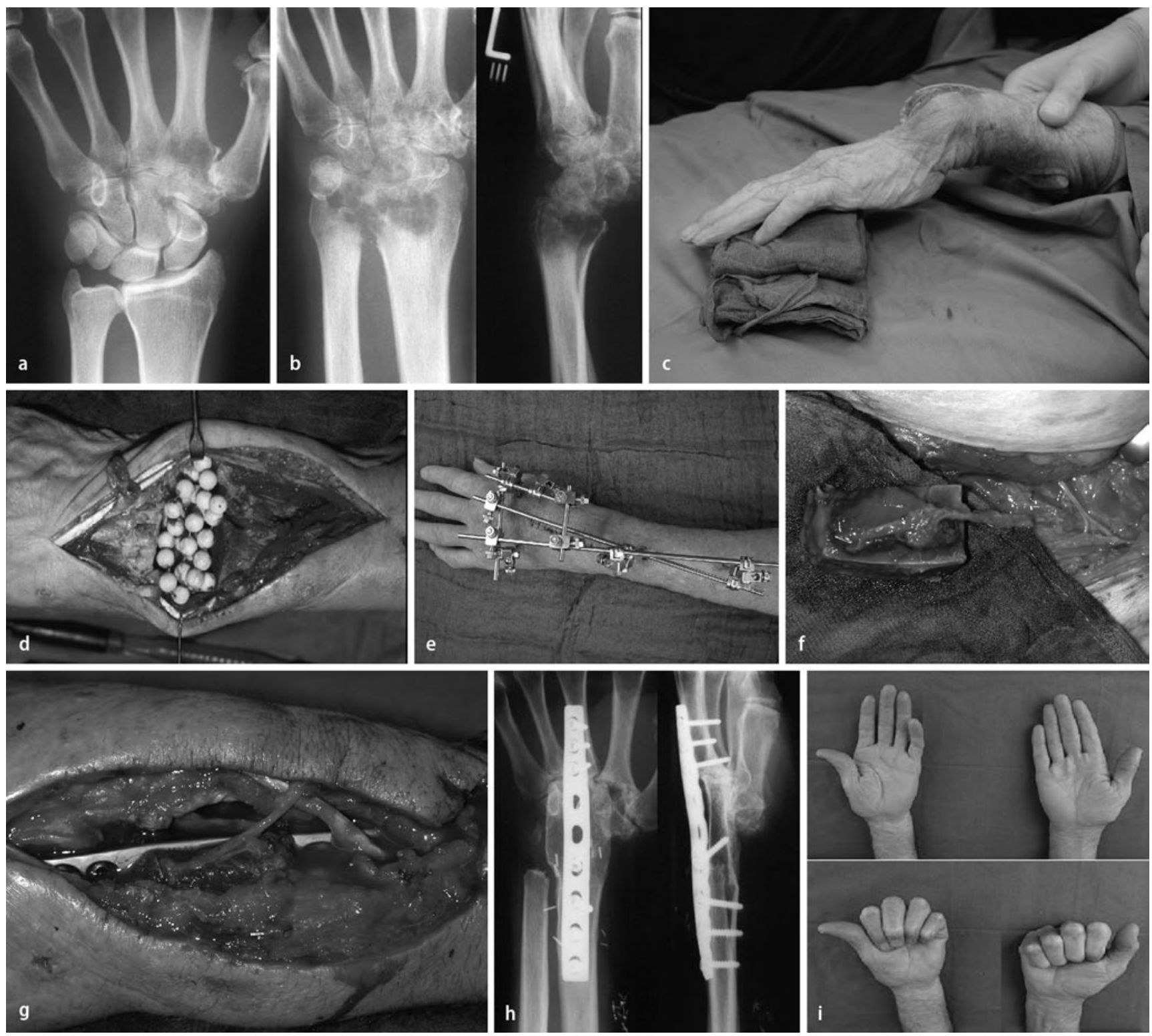

Abb. $1 \Delta$ Infektiöse Destruktion des Handgelenks nach Kortisoninjektion ins linke Daumensattelgelenk, a Röntgenaufnahme 6 Wochen nach der Injektion, CRP=116, Osteolyse im Bereich des CMC1- und CMC2-Gelenks, konservativ therapiert, b Destruktion des linken Handgelenks nach 6 Monaten, $C R P=2,1, \mathbf{c}-\mathbf{e}$ erste Operation mit Resektion des infizierten Knochens, Antibiotikaketteneinlage, Anlage eines temporären Fixateur externe, f,g zweite Operation nach 14 Tagen: Handgelenkarthrodese links mit freiem mikrovaskulärem Beckenkammspan von rechts, h,i Röntgenbefund und Funktion nach 3 Jahren (DASH 1,7; Kraft 26 kg links, entspricht 81\% der Gegenseite), CMC karpometakarpal, CRP C-reaktives Protein, DASH "disabilities of the arm, shoulder and hand"

len kann, sind nicht selten Lappenplastiken zur Weichteilrekonstruktion erforderlich. Die Ausheilung des Knocheninfekts erfordert oft eine mehrwöchige Antibiotikatherapie.

Entweder mit der Lappenplastik oder wenn reizlose Weichteilverhältnisse vorliegen und ein infektfreier Knochen wahrscheinlich ist, werden in einem oder mehreren Folgeeingriffen die fehlenden Strukturen (v. a. Knochen und Sehnen) wieder rekonstruiert [1]. Für die Knochenrekonstruktion sind nicht selten freie mikrovaskuläre Knochentransplantate erforderlich, die je nach Ausdehnung und Form von der Fibula, dem Beckenkamm, der Skapula oder dem medialen Femurkondylus gehoben werden.

\section{Fazit für die Praxis}

- Die Ursache einer Knocheninfektion an der Hand ist immer eine Inokulation von Bakterien, sei es durch eine offene Verletzung oder iatrogen durch eine Injektion oder Operation.

- Die Symptomatik einer Knocheninfektion an der Hand ist oft nur gering ausgeprägt, bei akuten Infekten ist 
die Operationsindikation schon bei dem bloßen Verdacht gegeben.

- Ein Keimnachweis gelingt meist nur aus Weichteil- und Knochenbiopsien, nicht aus einfachen Abstrichuntersuchungen.

- Die Therapie von Knocheninfekten beginnt mit dem radikalen operativen Débridement von Weichteilen und Knochen, ggf. Einlage von Antibiotikaträgern und temporärer Stabilisierung. Anschließend muss eine suffiziente Weichteildeckung erreicht werden.

- Der Knocheninfekt bedarf zur Ausheilung oft einer mehrwöchigen Antibiotikatherapie.

- Ein sekundärer Knochenaufbau mit interner Stabilisierung ist in der Regel spätestens nach 4 bis 6 Wochen möglich und erfordert bei ausgedehnten Infekten nicht selten die freie mikrovaskuläre Knochentransplantation von der Fibula, der Skapula, dem Beckenkamm oder dem medialen Femurkondylus.

\section{Korrespondenzadresse}

\section{Dr. B. Bickert}

Sektion Handchirurgie, Klinik für Hand-, Plastische und Rekonstruktive Chirurgie, BG-Klinik Ludwigshafen, Ludwig-Guttmann-Straße 13, 67071 Ludwigshafen

bickert@bgu-ludwigshafen.de

\section{Einhaltung ethischer Richtlinien}

Interessenkonflikt. B. Bickert gibt an, dass kein Interessenkonflikt besteht.

Dieser Beitrag beinhaltet keine Studien an Menschen oder Tieren.

The supplement containing this article is not sponsored by industry.

\section{Literatur}

1. Horch RE, Taeger CD, Steinau HU et al (2013) Osteomyelitis: Behandlungskonzepte aus Sicht der Plastischen Chirurgie. Chirurg 84:962-969

2. Hülsemann W, Habenicht R (2009) Schwere Nebenwirkungen nach Octenisept-Spülung von Perforationswunden im Kindesalter. Handchir Mikrochir Plast Chir 41:277-282

3. Klaeser B, Spanjol M, Krause T (2012) SPECT/CT-Infektdiagnostik am Skelett. Radiologe 52:615-620
Trauma Berufskrankh 2014 • 16[Suppl 3]:297-299 DOI 10.1007/s10039-014-2062-x

(c) Springer-Verlag Berlin Heidelberg 2014

B. Bickert

\section{Knocheninfekte an der Hand}

\section{Zusammenfassung}

Hintergrund. Knocheninfekte bei Erwachsenen werden immer durch eine Inokulation von Bakterien verursacht. Diese können nicht nur durch offene Verletzungen, sondern auch iatrogen ins Gewebe gelangen.

Diagnostik. Die klinische Beschwerdesymptomatik von Knochen- oder Gelenkinfekten ist meist nur gering ausgeprägt. Röntgenaufnahmen der Hand oder des Handgelenks sind bei entsprechendem Verdacht unabdingbar, bei unklaren, v. a. länger bestehenden Beschwerden können die Magnetresonanztomographie oder die SPECT/CT [Kombination von SPECT (,single photon emission computed tomography") und CT (Computertomographie)] hilfreich sein. Beweisend ist der Keimnachweis in Kombination mit dem histopathologischen Befund.

\section{Bone infections of the hand}

\section{Abstract}

Background. Bone infections in adults are always caused by invasion of bacteria into the tissue and can result not only from open injuries but can also have an iatrogenic origin. Diagnostics. The clinical symptoms of bone and joint infections are mostly weakly expressed. Radiological X-ray images of the hand and wrist are indispensible when an infection is suspected and magnetic resonance imaging (MRI) as well as the combination of single photon emission computed tomography and computed tomography (SPECT-CT) can be very useful especially in cases of longlasting complaints. Decisive are the detection and determination of the pathogen responsible in combination with the histological findings.
Therapie. Da der Schaden eines verschleppten Knochen- oder Gelenkinfekts gravierender ausfallen kann als der eines vielleicht unnötigen Eingriffs, ist die Operation bereits beim Verdacht auf einen Knochen- oder Gelenkinfekt indiziert. In vielen Fällen verbietet sich ein einzeitiges Vorgehen, in einem oder mehreren Eingriffen erfolgt das ausgiebige knöcherne Débridement mit temporärer Stabilisierung und Einlage eines antibiotikahaltigen Spacers, anschließend werden in einem oder mehreren Folgeeingriffen die fehlenden Strukturen rekonstruiert.

\section{Schlüsselwörter}

Knochen · Bakterielle Infektion · Hand · Débridement · Rekonstruktion
Therapy. Because the damage caused by a protracted bone or joint infection can have more serious consequences than a possibly unnecessary intervention, an operation is indicated even when a bone or joint infection is merely suspected. In many cases a one-sided approach is not possible; an extensive bony debridement with temporary stabilization and insertion of a spacer containing an antibiotic is performed in one or more interventions and defects are reconstructed in one or more subsequent interventions.

\section{Keywords}

Bones · Bacterial infections $\cdot$ Hand Debridement $\cdot$ Reconstructive surgical procedures

4. Meier R, Pillukat T (2011) Arthritis und Osteitis an der Hand. Handchir Mikrochir Plast Chir 43:131139

5. Zuluaga AF, Galvis W, Saldarriaga JG et al (2006) Etiologic diagnosis of chronic osteomyelitis. A prospective study. Arch Intern Med 166:95-100 